\title{
Metamaterial-dielectric photonics crystal waveguide structure
}

\author{
Hala J. El-Khozondar, ${ }^{1, ~ *, ~ R i f a ~ J . ~ E l-K h o z o n d a r ², ~ M o h a m m e d ~ M . ~ S h a b a t ~}{ }^{3}$ \\ ${ }^{1}$ Electrical Engineering department, Islamic University of Gaza, Gaza, Palestine \\ ${ }^{2}$ Physics department, Al-Aqsa University, Gaza, Palestine \\ ${ }^{3}$ Physics department, Islamic University of Gaza, Gaza, Palestine \\ Email address: \\ hkhozondar@iugaza.edu (H. J El-Khozondar), rifa20012002@yahoo.com (R. J El-Khozondar), shabat@gmail.com (M. M. Shabat)
}

\section{To cite this article:}

Hala J. El-Khozondar, Rifa J. El-Khozondar, Mohammed M. Shabat. Metamaterial-Dielectric Photonics Crystal Waveguide Structure. Optics. Special Issue: Optical Engineering \& Applications. Vol. 4, No. 1-2, 2015, pp. 1-4. doi: 10.11648/j.optics.s.2015040102.11

\begin{abstract}
Photonic crystals (PCs) are a revolutionary science that has a great impact on the field of photonics. In purpose of extend the applications of PCs, we introduce a new photonic structure which is composed of alternating lamellae of MTMs and Dielectric. The electromagnetic waves propagating through photonic crystal structures are numerically investigated. Results show improvement in the characteristics of the photonic crystal.
\end{abstract}

Keywords: Component, Formatting, Style, Styling, Insert

\section{Introduction}

Understanding of material properties helps to enhance their performance in practical applications. The progresses in metallurgy led to the development of various artificial materials with a fabulous range of mechanical properties. Semiconductors played an important role in modifying the conducting properties of materials, originating the revolution of transistors in electronics. To control optical properties of materials we are free to engineer the structure of materials that respond to light waves over a desired range of frequencies. The periodic microstructure of photonic crystal (PC) is similar to a crystalline lattice solid composed of a collection of atoms arranged in a regular repeated pattern. The periodic potential in the crystal is replaced by periodic dielectric constants in the photonic crystal. Mainly, we can build photonic crystals with photonic band gaps, prohibiting light from propagating in certain directions with specified frequencies [1].

Yablonovitch [2] had created a photonic band gap structure in which spontaneous emission can be severely inhibited in a way similar to the band gaps in semiconductors. The prevention of wave propagation in the forbidden gap has valuable applications as ideal reflective mirrors. Sigalas [3] studied waveguide structures in 3-dimensional metallic photonic band-gap materials, which can be used for the communication of different circuits in printed circuit boards. Similarly, Metamaterials (MTMS) are artificial materials, which have simultaneous negative permittivity and permeability. These materials are theoretically introduced by Veselago [4]. Later, MTMs are fabricated in the laboratory by Shelby [5] and Pendry [6]. These novel materials have unique properties, which make them valuable in practical applications such as sensors [7,8] and isolators [9].

The goal of this work is to study the application of metamaterials into photonic crystals. We proposed a structure consists of alternating lamellae of MTM and dielectric (SiO2). Next section is dedicated to the mathematical model. Results and discussion are given in section 3 followed by conclusion.

\section{Mathematical Model}

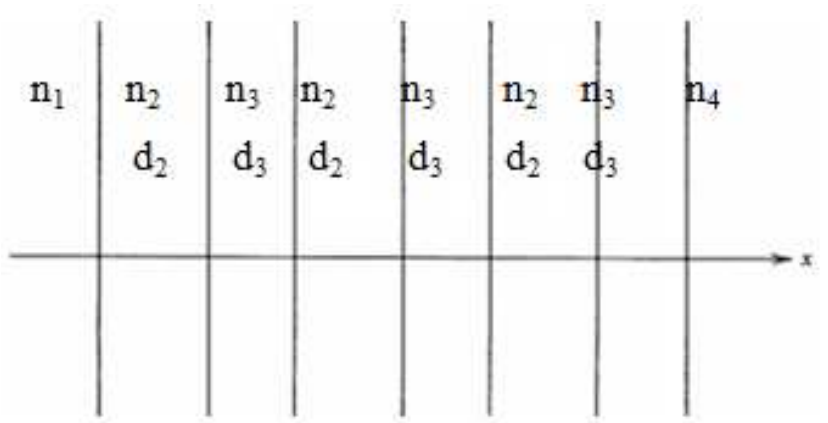

Figure 1. Proposed photonic structure: $n_{2}$ and $d_{2}$ are the refractive index and the thickness of the MTM, $n_{3}$ and $d_{3}$ are the refractive index and the thickness of the dielectric. 
Fig. 1 displays the proposed photonic crystal lamellar struture, which is constituted of MTM lamella having refractive indices $\mathrm{n} 2$ and thickness $\mathrm{d} 2$ alternating with dielectric lamella having refractive indices n3 and thickness $\mathrm{d} 3$. The bounded layers are air having refractive indices $n 1$ and $n 4$.

To determine the propagation of electromagnetic radiation in these media, we use the $2 \times 2$ array formulation. The electric field can be written as

$$
E=E(x) e^{i(\omega t-\beta z)}
$$

$$
E(x)=E_{R} e^{i k_{x} x}+E_{L} e^{-i k_{x} x}
$$

where $E_{R}$ and $E_{L}$ are constants, and $\pm k_{x}$ are the wave vector $x$ components for the waves travelling in the right and left directions.

The transfer matrix has the form [10]

$$
M=\left(\begin{array}{ll}
M_{11} & M_{12} \\
M_{21} & M_{22}
\end{array}\right)
$$

where

where $\beta$ is the $z$ component of the wave vector. We assume only s wave with $E / / y$ (TE) propagating in the xz plane with $E(x)$ can be written as

$$
\begin{gathered}
M_{11}=\frac{1}{2}\left[\left(1+\frac{n_{4} \cos \left(\theta_{4}\right)}{n_{1} \cos \left(\theta_{1}\right)}\right) \cos \phi_{2}+\left(\frac{n_{3} \cos \left(\theta_{3}\right)}{n_{1} \cos \left(\theta_{1}\right)}+\frac{n_{4} \cos \left(\theta_{4}\right)}{n_{3} \cos \left(\theta_{3}\right)}\right) i \sin \phi_{2}\right] \cos \phi_{1}+ \\
\frac{1}{2}\left[\left(\frac{n_{2} \cos \left(\theta_{2}\right)}{n_{1} \cos \left(\theta_{1}\right)}+\frac{n_{4} \cos \left(\theta_{4}\right)}{n_{2} \cos \left(\theta_{2}\right)}\right) \cos \phi_{2}+\left(\frac{n_{3} \cos \left(\theta_{3}\right)}{n_{2} \cos \left(\theta_{2}\right)}+\frac{n_{2} n_{4} \cos \left(\theta_{2}\right) \cos \left(\theta_{4}\right)}{n_{1} n_{3} \cos \left(\theta_{1}\right) \cos \left(\theta_{3}\right)}\right) i \sin \phi_{2}\right] i \sin \phi_{1}, \\
M_{12}=\frac{1}{2}\left[\left(1-\frac{n_{4} \cos \left(\theta_{4}\right)}{n_{1} \cos \left(\theta_{1}\right)}\right) \cos \phi_{2}+\left(\frac{n_{3} \cos \left(\theta_{3}\right)}{n_{1} \cos \left(\theta_{1}\right)}-\frac{n_{4} \cos \left(\theta_{4}\right)}{n_{3} \cos \left(\theta_{3}\right)}\right) i \sin \phi_{2}\right] \cos \phi_{1}+ \\
\frac{1}{2}\left[\left(\frac{n_{2} \cos \left(\theta_{2}\right)}{n_{1} \cos \left(\theta_{1}\right)}-\frac{n_{4} \cos \left(\theta_{4}\right)}{n_{2} \cos \left(\theta_{2}\right)}\right) \cos \phi_{2}+\left(\frac{n_{3} \cos \left(\theta_{3}\right)}{n_{2} \cos \left(\theta_{2}\right)}-\frac{n_{2} n_{4} \cos \left(\theta_{2}\right) \cos \left(\theta_{4}\right)}{\left.\left.n_{1} n_{3} \cos \left(\theta_{1}\right) \cos \left(\theta_{3}\right)\right) i \sin \phi_{2}\right] i \sin \phi_{1},}\right.\right. \\
M_{21}=\frac{1}{2}\left[\left(1-\frac{n_{4} \cos \left(\theta_{4}\right)}{n_{1} \cos \left(\theta_{1}\right)}\right) \cos \phi_{2}-\left(\frac{n_{3} \cos \left(\theta_{3}\right)}{n_{1} \cos \left(\theta_{1}\right)}-\frac{n_{4} \cos \left(\theta_{4}\right)}{n_{3} \cos \left(\theta_{3}\right)}\right) i \sin \phi_{2}\right] \cos \phi_{1}- \\
\frac{1}{2}\left[\left(\frac{n_{2} \cos \left(\theta_{2}\right)}{n_{1} \cos \left(\theta_{1}\right)}-\frac{n_{4} \cos \left(\theta_{4}\right)}{n_{2} \cos \left(\theta_{2}\right)}\right) \cos \phi_{2}-\left(\frac{n_{3} \cos \left(\theta_{3}\right)}{n_{2} \cos \left(\theta_{2}\right)}-\frac{n_{2} n_{4} \cos \left(\theta_{2}\right) \cos \left(\theta_{4}\right)}{n_{1} n_{3} \cos \left(\theta_{1}\right) \cos \left(\theta_{3}\right)}\right) i \sin \phi_{2}\right] i \sin \phi_{1}, \\
\frac{1}{2}\left[\left(\frac{n_{2} \cos \left(\theta_{2}\right)}{n_{1} \cos \left(\theta_{1}\right)}+\frac{n_{4} \cos \left(\theta_{4}\right)}{n_{2} \cos \left(\theta_{2}\right)}\right) \cos \phi_{2}-\left(\frac{n_{3} \cos \left(\theta_{3}\right)}{n_{2} \cos \left(\theta_{2}\right)}+\frac{n_{2} n_{4} \cos \left(\theta_{2}\right) \cos \left(\theta_{4}\right)}{n_{1} n_{3} \cos \left(\theta_{1}\right) \cos \left(\theta_{3}\right)}\right) i \sin \phi_{2}\right] i \sin \phi_{1} \\
M_{22}=\frac{1}{2}\left[\left(1+\frac{n_{4} \cos \left(\theta_{4}\right)}{n_{1} \cos \left(\theta_{1}\right)}\right) \cos \phi_{2}-\left(\frac{n_{3} \cos \left(\theta_{3}\right)}{n_{1} \cos \left(\theta_{1}\right)}+\frac{n_{4} \cos \left(\theta_{4}\right)}{n_{3} \cos \left(\theta_{3}\right)}\right) i \sin \phi_{2}\right] \cos \phi_{1}-
\end{gathered}
$$

with $k_{\alpha x}=\sqrt{\left(\frac{n_{\alpha} \omega}{c}\right)^{2}-\beta^{2}}, \beta=n_{\alpha} \frac{\omega}{c} \sin \left(\theta_{\alpha}\right), \alpha=1-4$.

$\phi_{1}=\frac{2 \pi \mathrm{d}_{2}}{\lambda} \mathrm{n}_{2} \cos \left(\theta_{2}\right)$, and $\phi_{2}=\frac{2 \pi \mathrm{d}_{3}}{\lambda} \mathrm{n}_{3} \cos \left(\theta_{3}\right)$.

The transmittance $(\mathrm{T})$ is given by

$$
T=|t|^{2}=\frac{n_{4} \cos \left(\theta_{4}\right)}{n_{1} \cos \left(\theta_{1}\right)}\left|\frac{1}{M_{11}}\right|^{2}
$$

The dispersion relation between the frequency $\omega$ and the forbidden band $\mathrm{K}$ for the special case $\beta=0$ (i.e. normal incidence) can be expressed by the following relation,

$$
\cos (K \Lambda)=\cos \left(k_{2} d_{2}\right) \cos \left(k_{3} d_{3}\right)-\frac{1}{2}\left(\frac{n_{3}}{n_{2}}+\frac{n_{2}}{n_{3}}\right) \sin \left(k_{2} d_{2}\right) \sin \left(k_{3} d_{3}\right)(5)
$$

where $K$ is the forbidden band, $k_{2}=(\omega / c) n_{2}, k_{3}=(\omega / c) n_{3}$, and $\Lambda$ is the lattice constant, $\Lambda=d_{2}+d_{3}$.

\section{Results and Discussions}

The dispersion relation (equation 10) is solved numerically. The transmittance (9) is then solved for different values of MTMs refractive index, $\mathrm{n} 2$. In all calculations, we have used 
the thickness of $\mathrm{SiO} 2$ layer is 340nm and the thickness layer of MTM is $4 \mathrm{~nm}$. The selected dielectric material refractive index $\mathrm{n} 3=1.54427$.

Figs. 2-8 illustrate the transmittance of s-waves as a function of the MTMs refractive index, $n 2$. It can be seen from these figures that the transmittance occurs in the range of visible light between $450 \mathrm{~nm}$ and $750 \mathrm{~nm}$ depending on the values of $\mathrm{n} 2$. Moreover, we noticed that transmittance might occur near the short IR for all case except at $n 2=-5$ and -8 .

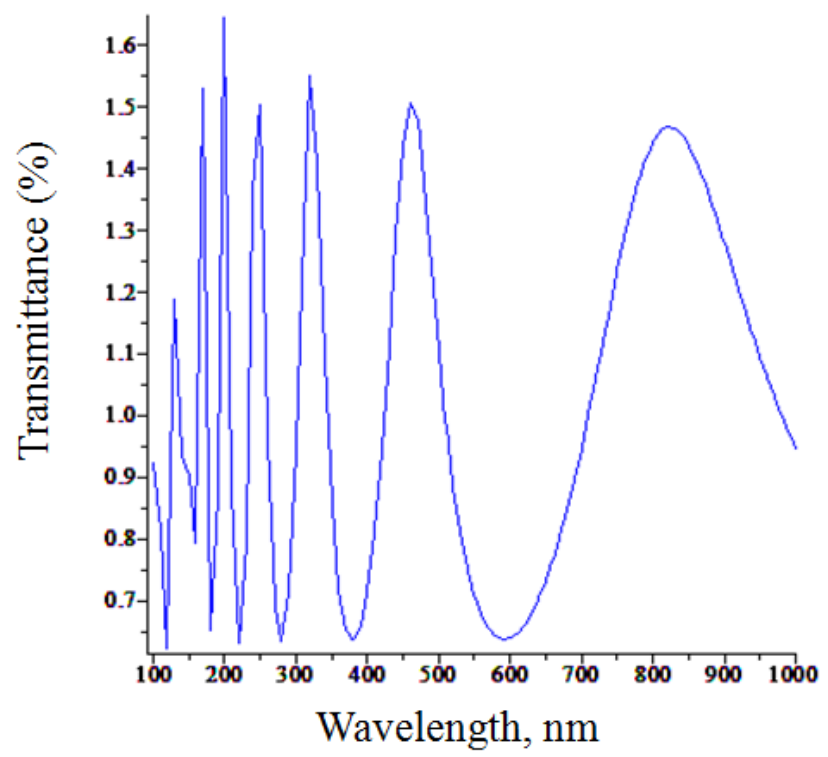

Figure 2. The transmittance of $s$-wave, $n_{2}=-1$. The waves propagate in the visible range around $470 \mathrm{~nm}$ and $800 \mathrm{~nm}$ and near the IR.

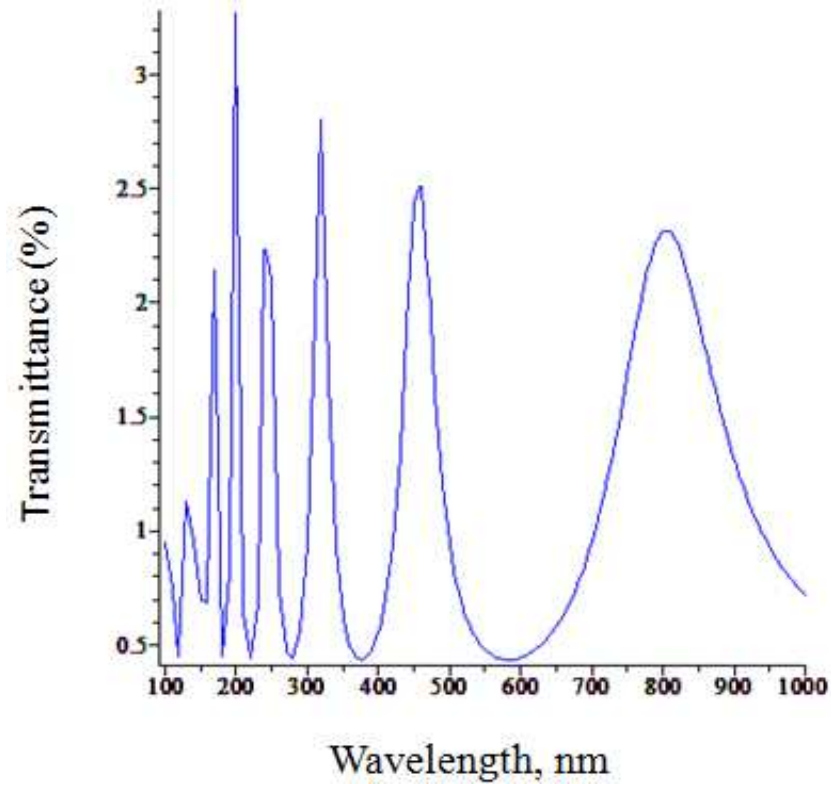

Figure 3. The transmittance of $s$-wave, $n 2=-2$. The waves propagate in the visible range around $470 \mathrm{~nm}$ and $800 \mathrm{~nm}$. and near the IR.

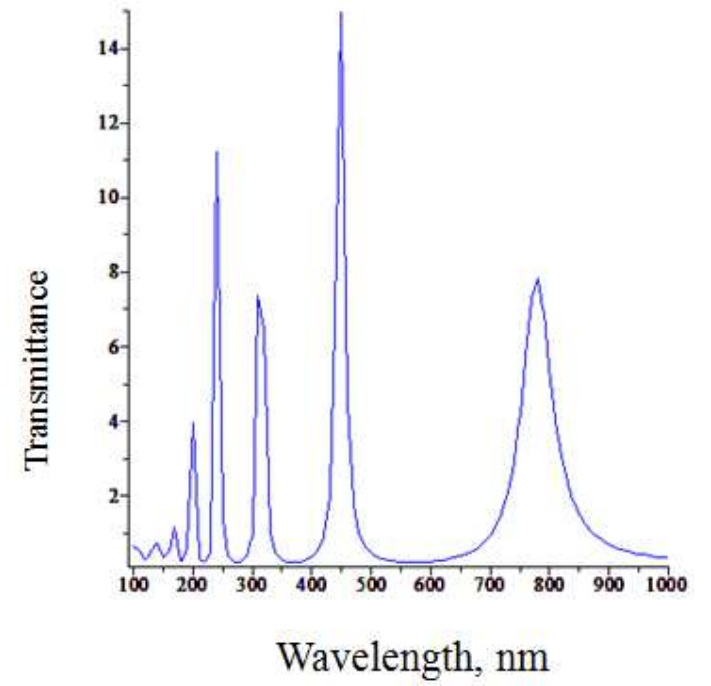

Figure 4. The transmittance of $s$-wave, $n 2=-4$. The waves propagate in the visible range around $440 \mathrm{~nm}$ and $800 \mathrm{~nm}$. and near the IR.

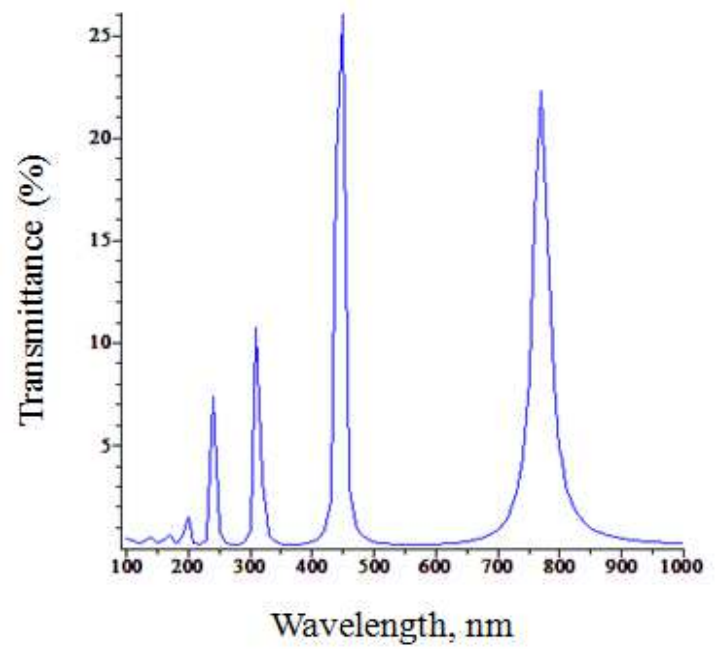

Figure 5. The transmittance of $s$-wave, $n 2=-5$. The waves propagate in the visible range around $450 \mathrm{~nm}$ and $780 \mathrm{~nm}$.

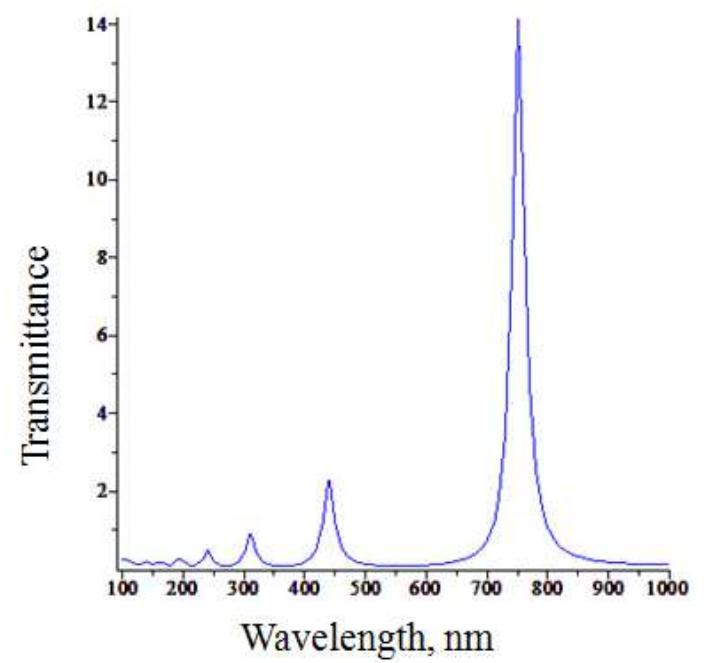

Figure 6. The transmittance of $s$-wave, $n 2=-8$. The waves propagate in the visible range around $450 \mathrm{~nm}$ and $750 \mathrm{~nm}$. 


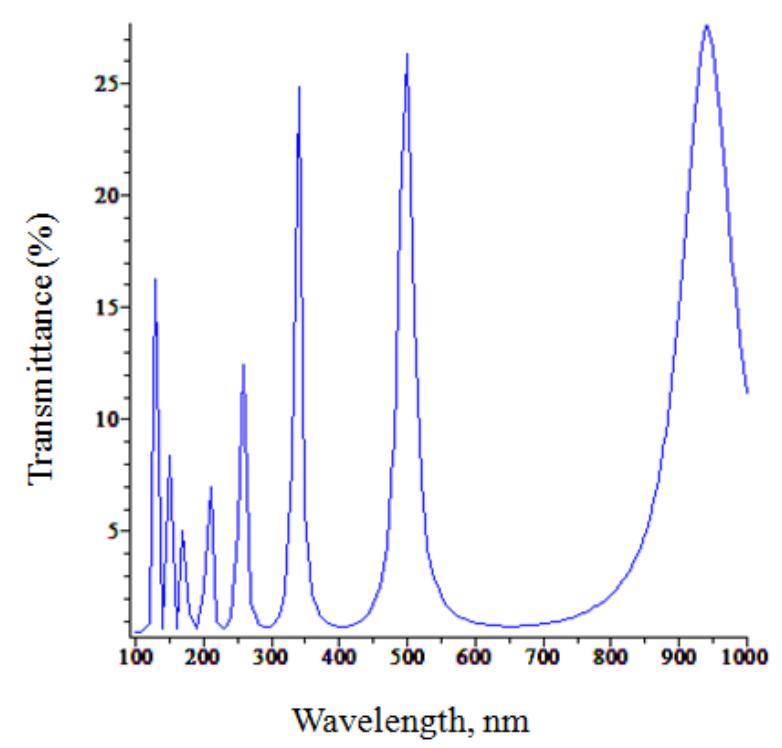

Figure 7. The transmittance of $s$-wave, $n 2=-1+i 2$. The waves propagate around $500 \mathrm{~nm}$ and near the IR.

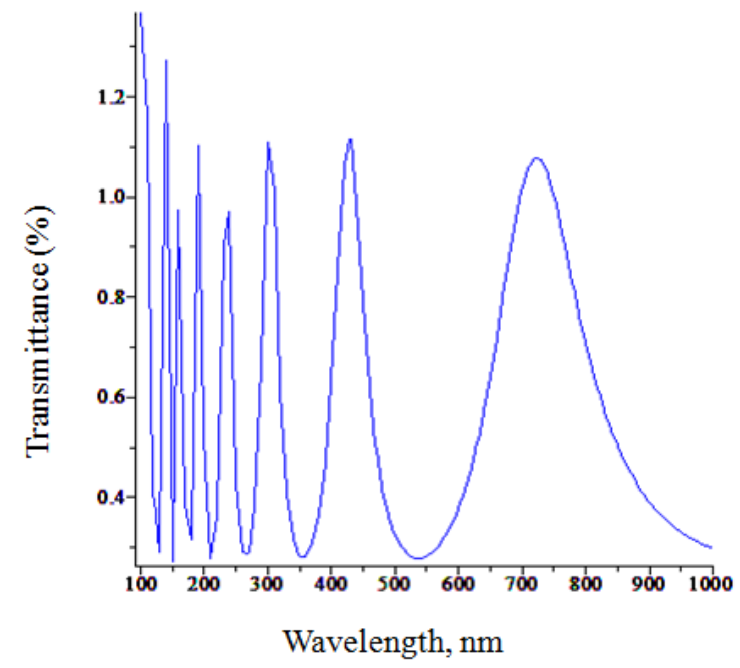

Figure 8. The transmittance of $s$-wave, $n 2=-1-i 2$. The waves propagate in the visible around $470 \mathrm{~nm}$ and $7500 \mathrm{~nm}$ and near the IR.

\section{Conclusions}

In the present work, we introduced PC consists of MTMs layer interchanged with dielectric layers. The structure works as a waveguide in the visible region near $450 \mathrm{~nm}$ and $750 \mathrm{~nm}$. Moreover, it allows transmission of short IR light.

\section{References}

[1] J. D. Joannopoulos, S.G. Johnson, J.N. Winn, R.D. Meade, Photonic Crystals: Molding the Flow of Light, 2nd Edition, Princeton University Press, 2008.

[2] E. Yablonovitch, Inhibited Spontaneous Emission in Solid-State Physics and Electronics, Phys. Rev. Lett., 58,2059-2062,1987.

[3] M. M. Sigalas, R. Biswas, K. M. Ho, and C. M. Soukoulis, Waveguides in three-dimensional metallic photonic band-gap materials, Phys. Rev. B, 60, 4426- 4429, 1999.

[4] V. G.Veselago, The electrodynamics of substances with simultaneously negative values of $\varepsilon$ and $\mu$, Soviet Physics, 10, 509-514, 1968.

[5] R. A. Shelby, D. R. Smith, and S. Schultz, Experimental verification of negative index of refraction, Science, 292 77-79, 2001.

[6] J. B. Pendry, Negative refraction makes a perfect lens, Phys. Rev. Lett., 85, 3966-3969, 2000.

[7] H. J. El-Khozondar, R. J. El-Khozondar, S. Zouhdi, Surface waves at the interface between tunable LC-MTMs and nonlinear media, Appl. Phys. A, 109, 865-867, 2012.

[8] H. J. El-Khozondar, M. Müller, R. J. El-Khozondar, M. Shabat, A. Koch, Sensitivity of double-negative metamaterial optical sensor, Int. J. Pure Appl. Sci. Technol., 11, 29-39, 2012.

[9] H. El-Khozondar, R. El-Khozondar, M. Shabat, Characteristics of Magetooptical Integrated Metamaterials Isolator, Int. J. Mod. Phys. B, 23, 4675-4683, 2009.

[10] P. Yeh, Optical waves in layered media, Wiley \& Sons, USA, 1988. 